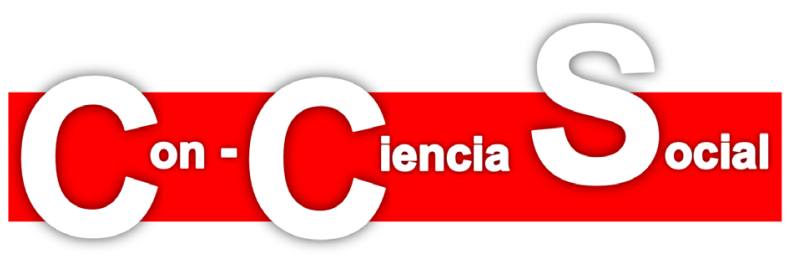

Ciencia privatizada en América Latina

\title{
Privatized science in Latin America
}

\author{
Cecilia Rikap \\ Universidad de Buenos Aires / CEPED, IRD/Université de Paris \\ ceciliarikap@gmail.com
}

\author{
Judith Naidorf \\ Universidad de Buenos Aires \\ judithnaidorf@gmail.com
}

Recibido en noviembre de 2019

Aceptado en enero de 2020

DOI:10.7203/con-cienciasocial.3.16790

\section{RESUMEN}

En regiones como América Latina, la mercantilización de la ciencia involucra su subordinación a la producción de innovaciones a nivel global que refuerzan las estructuras actuales de poder y el lugar dependiente de la región. Para dar cuenta de estas especificidades se propone el concepto de ciencia privatizada entendida como la ciencia que por medio de copiar agendas y prioridades exógenas al contexto de creación del conocimiento y a las necesidades sociales (y aunque sus resultados sean públicos), igualmente beneficia la acumulación de ganancias privadas. Lo hace sin que quede rédito para las instituciones productoras de ese conocimiento, ni tampoco por lo tanto para sus países de origen. El trabajo muestra dimensiones concretas de la ciencia privatizada, a saber: la transferencia ciega de conocimiento, la orientación de la agenda de investigación a prioridades de empresas o de organismos internacionales extranjeros, o copiando de manera acrítica la agenda internacional mainstream, y el peso del oligopolio editorial internacional. Estas dimensiones calan hondo en la región dado que el artículo publicado en revistas internacionales con referato se privilegia como criterio de evaluación. De modo que el trabajo concluye realizando propuestas de indicadores alternativos para la evaluación del trabajo científico y tecnológico público.

Palabras clave: mercantilización, ciencia privatizada, transferencia ciega de conocimiento, agendas de investigación, oligopolio editorial, América Latina.

\begin{abstract}
In regions such as Latin America, the commodification of science involves its subordination to the production of innovations at the global level that reinforce current power structures and the dependent place of the region. To account for these specificities, the article introduces the concept of privatized science. It is understood as science that, by copying exogenous agendas and priorities alien to the context of knowledge creation and social needs (and even if its results are public), benefits the accumulation of private profits without any revenue being left for the institutions producing that knowledge, nor therefore for their countries of origin. The article shows concrete dimensions of privatized science, namely: blind transfer of knowledge, the orientation of the research agendas to the priorities of foreign companies or international organizations, and/or to mainstream international agenda, and the relevance of the international editorial oligopoly. These dimensions penetrate deeply into the region given that papers published in peer-review international journals are the privileged evaluation criteria. Thus, this article ends by suggesting alternative indicators for the evaluation of public science and technology research.
\end{abstract}

Keywords: commodification, privatized science blind knowledge transfer, research agendas, editorial oligopolies, Latin America

\section{Referencia}

Rikap, C. y Naidorf, J. (2020). Ciencia privatizada en América Latina. Con-Ciencia Social (segunda época), 3, 57-76. DOI:10.7203/con-cienciasocial.3.16790 


\section{INTRODUCCIÓN}

La mercantilización y privatización del conocimiento implica una transformación más compleja y amplia que el reconocimiento de la autorización y fomento a patentar los resultados de las investigaciones académicas. Significa también que el conocimiento generado en ámbitos públicos con financiamiento privado conlleva cláusulas de confidencialidad que limitan su libre circulación, así como la estimulación a seleccionar temas de investigación "rentables" y la valoración del trabajo académico según criterios empresariales (Naidorf, 2009). Implica, asimismo, la combinación de presiones del mercado y políticas científicas activas de orientación de las investigaciones por parte del Estado. En regiones como América Latina, la mercantilización de la ciencia involucra también su subordinación a la producción de innovaciones a nivel global que refuerzan las estructuras actuales de poder y el lugar dependiente de la región.

En este escenario, nos proponemos mostrar que las tendencias a la mercantilización y privatización de la ciencia pública en Latinoamérica no se limitan a la promoción de la colaboración entre academia e industria. De hecho, la misma ha tenido un limitado efecto en las culturas académicas locales especialmente en las áreas de ciencias sociales, humanidades, ciencias exactas y naturales, y en las ciencias de la salud (Rikap \& Harari-Kermadec, 2019). Son en cambio otros criterios heterónomos los que ejercen mayor presión. En particular, destacamos la importación acrítica de la agenda internacional y el impacto programático y performativo de los criterios de evaluación principalmente asentados en la cientometría y el circuito mainstream de publicaciones.

En comparación con los países centrales, las empresas que operan en la región no invierten significativamente en I+D (Albornoz, Barrere y Sokil, 2017; Arocena, Göransson, \& Sutz, 2015), reforzando una estructura productiva históricamente rezagada (Iñigo Carrera, 2007; Llomovatte, 2006). Asimismo, el discurso asociado a la colaboración universidad-empresa ha servido para justificar los recortes del presupuesto público y ha sido presentado como la panacea frente a un problema de larga data.

En tiempos de aumentos de la inversión pública en ciencia y tecnología (principalmente durante la primera década del $2000^{1}$ ) las políticas científicas han

\footnotetext{
${ }^{1}$ El aumento en la inversión pública en C\&T ha sido desigual pero tendiente al alza en países como Argentina, México, Brasil, Paraguay, Bolivia, Honduras y Guatemala. Al respecto hemos llevado a cabo
} 
continuado con el plan de promover dicha asociación a través de la creación de instrumentos de promoción del vínculo con empresas. Sin embargo, no ha sido significativa la inversión privada en ciencia, aunque sí la inversión pública en ciencia privatizada. Por otra parte, el discurso mercantil resurge con nuevas aristas y asociado a nuevos recortes de la mano de una agenda neoliberal que se impulsó con fuerza a partir de 2015 en el Cono Sur.

A fin de dar cuenta de las especificidades de una región dependiente en el proceso global de mercantilización y privatización del conocimiento, el resto de este artículo se estructura de la siguiente manera. En el próximo apartado damos cuenta de la relevancia de las universidades como ámbito de producción de conocimiento en América Latina. Seguidamente, desarrollamos el concepto de ciencia privatizada. Luego, el resto de este trabajo aborda, preliminarmente, distintas dimensiones de la ciencia privatizada en la región, y cierra con aportes concretos para la revisión de los criterios de evaluación de la ciencia pública. Estos criterios alternativos se proponen limitar la excesiva ponderación que recibe actualmente la producción científicotecnológica medida a través de publicación en revistas con referato que las agencias de financiamiento y evaluación sobreponderan. Es un criterio que poco reconoce la existencia de un mercado editorial. Asimismo, desconoce e implícitamente refuerza la orientación de la producción de conocimiento a la agenda de cada disciplina en los países centrales, la cual está fuertemente influenciada por monopolios intelectuales globales.

\section{LAS UNIVERSIDADES COMO ÁMBITO PRIVILEGIADO PARA LA PRODUCCIÓN DE CONOCIMIENTO EN LATINOAMÉRICA}

Las universidades públicas son el principal ámbito de producción de conocimiento y el lugar de trabajo privilegiado de los investigadores en los países en desarrollo (OECD, 2012). En América Latina, este lugar central se explica por las capacidades de las universidades públicas, pero también por la reducida investigación y desarrollo (I+D) realizada y financiada por empresas privadas, que en la región suelen limitarse a ser demandantes de asistencia técnica (Albornoz, Barrere y Solki, 2017; Arocena \& Sutz, 2010).

un estudio comparado entre investigadores de los nombrados países compilados en la Revista Cubana de Educación Superior, vol. 34, núm. 1. 
En virtud de lo anterior, la mercantilización y privatización del conocimiento se manifiestan con características específicas y diferenciadas respecto de las usuales en otras latitudes. Aquí, el patentamiento de los conocimientos no es relevante, los contratos con empresas son en su mayoría para la realización de tareas rutinarias (asistencias técnicas o asesorías complejas que no suponen investigación creativa), y el peso de los recursos privados en el presupuesto público de las universidades nacionales es bajo.

Aunque el sistema de evaluación del desempeño de los investigadores ha venido dando señales en torno a la ponderación positiva de los denominados STAN (Servicios Tecnológicos de Alto Nivel), en la cultura académica de los científicos y científicas no ha calado como práctica frecuente. Asimismo, las patentes procuran aun con evidentes dificultades de gestión convertirse en un indicador relevante de buenas prácticas académicas, pero localmente lo logran con dificultad.

A pesar de que los ingresos por este tipo de servicios y las patentes locales no son significativos, sí vale destacar las señales que emiten los sistemas de evaluación en el fomento de dichas actividades. Al respecto la adaptación de las y los investigadores a los criterios de evaluación es una tendencia creciente a nivel mundial.

Adicionalmente a la emergencia de políticas que fomentan prácticas mercantiles que no se concretan pero que igualmente moldean las lógicas de investigación de ciertos grupos de investigadores, nos interesa profundizar sobre un proceso que afecta a toda la comunidad académica de la región. Proceso que la afecta más allá de los distintos posicionamientos respecto a las políticas explícitas de ciencia mercantilizada. Nos referimos a formas específicas en las que la investigación periférica pública contribuye al rédito privado, en general, de empresas de capitales de países centrales y que frecuentemente pasan desapercibidas por los investigadores e investigadoras. Hacia allí avanzamos con la noción ciencia privatizada.

\section{LA CIENCIA PRIVATIZADA}

La categoría de ciencia privatizada da cuenta de las tendencias a copiar agendas y prioridades exógenas al contexto de creación del conocimiento y a las necesidades sociales. También es ciencia privatizada aquella que iguala calidad a indexadores internacionales inmersos en un mercado editorial donde las y los investigadores pagan por publicar, y luego sus universidades pagan por el acceso a dicho conocimiento 
publicado. Asimismo, asociamos la ciencia privatizada con la orientación mercantil de la investigación que se esfuerza más por la cura que por la prevención de los males que aquejan a las y los pobladores de Latinoamérica.

Aunque la ciencia y sus resultados formalmente permanezcan en el ámbito público, tal como funciona actualmente la que calificamos de ciencia privatizada en América Latina, se está contribuyendo a la apropiación de ganancia por parte de ciertas empresas. Estas son generalmente transnacionales que declaran sus ganancias en países centrales o paraísos fiscales. A nivel global, se ha puesto en evidencia que aquellas empresas que concentran activos intangibles son también aquellas que tienen mayores ganancias (Orhangazi, 2018). Entre los activos intangibles, los derechos de propiedad intelectual han sido destacados a la hora de hacer referencia a la emergencia de un capitalismo de monopolios intelectuales (Durand \& Milberg, 2019; Pagano, 2014; Rikap, 2018).

En este proceso de sistemática acumulación privada de innovaciones, la ciencia producida en los ámbitos públicos resulta un insumo valioso por su calidad y porque los costos de su producción son en parte asumidos por el Estado. Las empresas hacen uso de estos beneficios con el objetivo de potenciar su producción de innovaciones y con ella la obtención de mayores ganancias. En este contexto, si bien los vínculos formales de transferencia tecnológica se refuerzan, en este tipo de interacción generalmente se privilegia a las instituciones líderes de países centrales, tal como identificó Rikap (2019) para el caso de la industria farmacéutica.

Cuando enfocamos nuestra atención en países periféricos como los latinoamericanos, los mecanismos privilegiados para extracción de rentas intelectuales de la investigación pública tienen un carácter menos explícito, dado que no pasan por las oficinas de transferencia tecnológica. De hecho, dentro de los recursos privados (llamados localmente recursos propios), en las instituciones de la región predominan los ingresos asociados a la enseñanza, en particular los aranceles. Allí donde la enseñanza pública de grado es gratuita, los ingresos por aranceles de cursos de posgrado, seguidos por las mencionadas asistencias técnicas, se destacan (Dávila, 2006; Juarros, 2006; Rikap, 2017; Riquelme y Langer, 2013). De modo que es necesario observar otros indicadores para complementar este panorama y así comprender las múltiples dimensiones de la privatización y mercantilización del conocimiento en la actualidad. 
¿De qué maneras contribuye la ciencia producida en América Latina a la generación de mayores ganancias, principalmente de empresas de países centrales? Afirmamos aquí que lo hace a través de la ciencia privatizada cuyas múltiples dimensiones incluyen las siguientes: a) la transferencia ciega de conocimiento que procura mesurar el impacto de la investigación latinoamericana en las patentes internacionales (generalmente tramitadas fuera de la región y cuyos beneficios no retornan); b) la reorientación de la agenda de investigación en la búsqueda (típicamente infructuosa) de comercialización de resultados exitosos; c) la adaptación de agendas de investigación nacionales o regionales a las prioridades de la agenda internacional de la disciplina -principalmente en el caso de las ciencias exactas, naturales, medicina, de la computación, entre otras- que responden cada vez más a las prioridades de la investigación privada y que se evidencian, entre otros indicadores, por medio de la co-publicación con empresas; d) los subsidios a investigación otorgados por empresas privadas, organismos internacionales o de ciencia y técnica de países centrales; y e) el peso de las editoriales privadas en el acceso a la investigación publicada y a la publicación, entre otros.

Estos indicadores pintarán un cuadro más complejo en el cual el episodio regional de las tendencias globales incluye un proceso de extractivismo del conocimiento. El mismo beneficia a las grandes empresas líderes globales, al tiempo que refuerza la dependencia académica y económica de Latinoamérica.

\section{MECANISMOS POR LOS QUE OPERA LA CIENCIA PRIVATIZADA}

\section{LA TRANSFERENCIA CIEGA DE CONOCIMIENTO}

La cita de publicaciones científicas en las solicitudes de patentes, llamada por Codner, Becerra y Díaz (2012) transferencia ciega de tecnología, es una forma de examinar la contribución de la ciencia a la producción de innovaciones. Esta contribución escapa a las oficinas de transferencia tecnológica, y también es ignorada por los propios investigadores e investigadoras y sus evaluadores y evaluadoras. Por eso, su existencia no puede ser relevada por medio de entrevistas o encuestas. En nuestro caso, preferimos llamar a este vínculo transferencia ciega de conocimiento ya que las publicaciones citadas pueden estar lejos de las contribuciones tecnológicas, pero igualmente representan una transferencia de conocimientos. Aunque este es un indicador menos frecuente, ha sido reconocido como valioso para medir la 
contribución de la ciencia pública a las innovaciones privadas (Ahmadpoor \& Jones, 2017; Codner, Becerra, \& Díaz, 2012; Codner \& Perrota, 2018; Wang \& Li, 2019).

A los fines de evidenciar la relevancia de este indicador, podemos comparar la transferencia ciega de conocimientos de la Universidad de Buenos Aires (UBA) en el área de farmacia y farmacología con universidades mundialmente reconocidas como líderes en materia de contribución a la producción de innovaciones. Entre 1998 y 2017 , 57 de las 1.232 (4,6\%) publicaciones científicas con al menos un autor o autora de la UBA registradas en Web of Science fueron citadas en patentes internacionales correspondientes a tres oficinas de patentes: la UPSTO de Estados Unidos, la Oficina Europea de Patentes y la Oficina Coreana de Propiedad Intelectual. Tijssen (2019) ha observado recientemente que el 3,4\% del total de las publicaciones de Harvard han sido citadas en patentes internacionales entre 2006 y 2015. Las transferencias ciegas de conocimiento de otras instituciones líderes más enfocadas a la investigación aplicada o tecnológica, como la Facultad de Medicina de Hannover $(3,1 \%)$, el MIT $(4,6 \%)$ o la ETH Zurich (1,9\%), están en línea con los resultados obtenidos para la UBA en farmacia y farmacología.

Lo que pretendemos mostrar aquí es que la región no se hace de réditos económicos asociados a dicha actividad creativa. Este indicador tampoco es considerado en las evaluaciones del desempeño académico. Así, se subvalora el impacto de la producción local en producciones patentadas en los países centrales. Considerar este indicador alternativo apunta a poner de manifiesto que, aunque las empresas de capital que operan en la región efectivamente no demanden transferencia de tecnología a la universidad, las empresas de capital de países centrales sí hacen uso de los resultados obtenidos, al menos por investigadores e investigadoras de ciertas universidades de la región.

\section{LA REORIENTACIÓN DE LA AGENDA DE INVESTIGACIÓN EN LA BÚSQUEDA (TÍPICAMENTE INFRUCTUOSA) DE COMERCIALIZACIÓN DE LOS RESULTADOS}

La cultura académica de Latinoamérica valora y responde a criterios de autonomía y libertad de elección de los temas a investigar. En la región, la autonomía como principio estructurador de la universidad encuentra un antecedente temprano en la Reforma Universitaria de Córdoba de 1918, profundizándose luego en el tiempo y en el espacio. Contribuyó a generar en las universidades de la región reticencias y desconfianzas a la hora de relacionarse con gobiernos y empresas. La historia de 
luchas contra las intervenciones tanto de gobiernos de facto como democráticos reforzó aquellas reticencias (Buchbinder, 2005; Juarros y Martinetto, 2008; Sutz, 2000). Quienes recibían fondos de gobiernos extranjeros, fundaciones (Ford, Rockefeller, Carnegie) o empresas privadas eran acusados de reproductores de la cultura hegemónica, o de quedar sus investigaciones subordinadas a los requerimientos privados (Bekerman, 2010; Naidorf, 2006; Navarro y Quesada, 2010).

No obstante esta tradición, las restricciones económicas que impactaron en la investigación científica y las tendencias internacionales que buscaron asociar ciencia académica con innovación llevaron a las y los científicos a intentar incrementar sus recursos mediante la comercialización de los resultados de sus investigaciones. A partir de la década del 80 se crearon instrumentos de política científica que procuraron acompañar este proceso y motivar a los equipos de investigación a direccionar sus agendas hacia temas potencialmente rentables en términos de transferencia. La creación de la Oficina de Transferencia de Tecnología del Consejo Nacional de Investigaciones Científicas y Tecnológicas (CONICET) en Argentina en 1984 es un ejemplo de los intentos por fomentar esta nueva tendencia. En toda América Latina se crearon leyes de innovación y una batería discursiva que procuraba romper con la tradición autonomista y buscar en la empresa un aliado con el que paliar la desfinanciación pública en ciencia recrudecida en los 90 de la mano de las políticas neoliberales que imperaron en el continente. En este marco, aquel histórico rechazo a toda potencial restricción a la autonomía universitaria fue parcialmente reemplazado por colaboraciones más fluidas con otros actores, tanto públicos como privados (Arocena \& Sutz, 2001).

Las empresas recibieron algunos estímulos fiscales para llevar a cabo estas tareas, pero no lograron repercutir en una industrialización basada en el conocimiento en un contexto donde la dependencia (tecnológica, financiera, etc.) resultaba ser el destino para la región. Si bien permanece en el discurso la necesidad de ajustar las agendas a temas que pudieran generar la comercialización de los conocimientos, ha sido demostrado el escaso impacto de este tipo de acciones.

Rikap y Harari-Kermadec (2019) proveen evidencia empírica acerca de la transferencia de tecnología a la industria en Argentina afirmando que esta no tiene impacto en los criterios de evaluación del desempeño de las y los académicos. En el 
año $2012^{2}$ se inició un espacio de discusión sobre criterios de evaluación del que participaron todos los organismos de Ciencia y Técnica del país que fue luego discontinuado en el tiempo.

La transferencia no es considerada dentro de los criterios de evaluación de los investigadores universitarios (Beigel, 2017; Unzué, 2013). Además, dado que la cantidad de artículos científicos en revistas con referato sigue siendo el criterio principal a la hora de evaluar el ingreso de los investigadores al CONICET y para lograr promociones, la publicación en revistas con alto factor de impacto persiste como la práctica más común (Beigel, 2017), independientemente de las colaboraciones con la industria. Hasta cierto punto, este resultado muestra una incoherencia interna entre el aparente fomento a las colaboraciones público-privadas, y criterios de evaluación que privilegian las publicaciones científicas. El intento de incluir la transferencia de conocimientos como criterio de evaluación no parece haber afectado significativamente el predominio de la publicación de artículos.

Sin embargo, Rikap y Harari-Kermadec (2019) hallaron una correlación entre las líneas estratégicas de investigación definidas en Argentina y el impacto sobre la trasferencia de tecnología a la industria. Estas áreas, en particular las que no conciernen a las ciencias sociales y humanas, están en línea con las necesidades de la industria y privilegian la investigación aplicada. Los temas considerados estratégicos fueron enunciados y reflejados en políticas científicas, tanto por gobiernos neoliberales como por los que procuraron ser populares. Si bien la privatización y mercantilización de la academia es compatible incluso con el pensamiento desarrollista que coloca al conocimiento como un bien vendible, ésta adquiere matices en una práctica no resuelta entre criterios endogámicos (la ciencia para el ensanchamiento de las fronteras del conocimiento y de las disciplinas) y criterios amplios de relaciones y respuestas en el marco de una ciencia pensada en y con el entorno.

Ahora bien, la orientación de la agenda de investigación en función de líneas consideradas estratégicas que buscan responder a necesidades de la industria moldea las investigaciones públicas en función de necesidades privadas. Otro mecanismo específico de orientación de las investigaciones se produce cuando

\footnotetext{
2 Al respecto se puede observar reflejada esta discusión en los medios de comunicación: http://www.pagina12.com.ar/diario/universidad/index-2012-10-05.html, y principalmente en los Documentos I y II que enuncian los acuerdos arribados por la Comisión Asesora sobre Evaluación del Personal Científico Tecnológico: https://www.argentina.gob.ar/ciencia/banco-pdts
} 
investigadores del sector público trabajan en conjunto con empresas en proyectos de investigación. No se trata aquí de una transferencia de tecnología, sino del desarrollo conjunto de investigaciones. Una forma de evidenciar esta práctica es la copublicación con empresas (Confraria \& Vargas, 2019). Esta práctica ha sido identificada como uno de los canales más importantes de transferencia de conocimientos en América Latina (Dutrénit \& Arza, 2010) y suele pasar inobservado en los estudios que se concentran en indicadores que miden el rédito económico de las vinculación universidad-empresas para la primera. Ello, pues no producen ningún beneficio económico para la universidad, mientras cabe esperar que los resultados alcanzados se inserten en redes de innovación de la empresa co-autora y así, eventualmente, sirvan de base para innovaciones que generen un mayor beneficio privado.

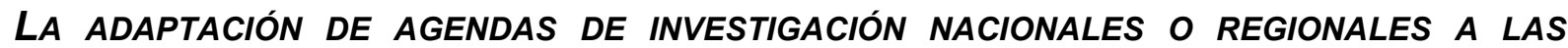
PRIORIDADES DE LA AGENDA INTERNACIONAL DE LA DISCIPLINA

Los autores que se enmarcan en la tesis de dependencia académica examinan la investigación científica de los países periféricos, analizando cómo adoptan la agenda de investigación de los países hegemónicos (Beigel, 2010). Originalmente, esta tesis refiere a la constatación de que las y los investigadores de países no hegemónicos usualmente ocupan posiciones subordinadas en la división global del trabajo científico. Sin embargo, se han señalado las limitaciones de medir la "ciencia mundial" basada en las bases de datos de publicaciones dominadas por países centrales (Vessuri, 1987). Desde una perspectiva más crítica, la investigación periférica no es unilateralmente subordinada a la agenda científica de los países centrales (Beigel, Gallardo, \& Bekerman, 2018). Beigel et al. (2018) argumentan que la periferia no está representada por disciplinas homogéneas que se caracterizan colectivamente por su dependencia con los centros de producción de conocimiento. Por el contrario, la dependencia se desarrolla por medio de mecanismos más complejos en los cuales la periferia no es mera importadora pasiva de conocimiento.

De este modo, la adaptación de agendas de investigación, tanto frente a las presiones de empresas (locales y multinacionales) como frente a las líneas de investigación que se imponen en países centrales y que frecuentemente responden a las necesidades de sus empresas (muchas de ellas multinacionales), tiene que entenderse como un proceso dinámico. En dicho proceso, las presiones del sector 
privado y de la globalización del conocimiento se enfrentan a comunidades científicas con tradición, lógicas, prácticas y objetivos políticos específicos que muchas veces están en contradicción con aquellas tendencias. El interjuego creativo se evidencia en producciones originales que escapan al circuito mainstream de publicación, aunque la evaluación académica se encuentre varios pasos por detrás de la rica producción de conocimiento en la región.

\section{LOS SUBSIDIOS A INVESTIGACIÓN OTORGADOS POR EMPRESAS PRIVADAS Y ORGANISMOS INTERNACIONALES O DE CIENCIA Y TÉCNICA DE PAÍSES CENTRALES}

El dilema sobre la injerencia en la definición de agendas de investigación en América Latina por parte de organismos internacionales y empresas multinacionales se inicia en los '50 con la fundación Ford, Rockefeller y Carnegie, y luego con organismos que van desde la UNESCO al Banco Mundial. Estos instalaron sedes en la región desde donde impulsaron temas que fueron objeto de indagación de la ciencia latinoamericana (Naidorf, 2009).

Desde Estados Unidos se elaboraron el Fullbright Program y el US.AID, entre otros. En Europa, por ejemplo, se crearon la Swedish Agency for Research Cooperation en Suecia, y la CNRS-CCFD en Francia. El objetivo más o menos explícito tanto de fundaciones como de organismos internacionales que operaron desde entonces en la región ha sido direccionar las políticas académicas y con ello el tipo y fin del conocimiento producido (Navarro y Quesada, 2010).

A nivel global, en relación a las dimensiones que atañen a la orientación de las agendas de investigación, la relación periférica y la mediatización tecnológica continuaron colocando a América Latina como satélite de las agendas definidas en los países centrales. Incluso la cientometría y el acoplamiento a los estándares exógenos de supuesta calidad en función de factores de impacto de las revistas en donde se publica, han calado con desiguales niveles de conciencia en las comunidades académicas.

\section{EL PESO DE LAS EDITORIALES PRIVADAS EN EL ACCESO A LA INVESTIGACIÓN PUBLICADA Y A}

\section{LA PUBLICACIÓN}

El negocio de las editoriales tiene, al menos, dos dimensiones: la del oligopolio mundial de editoriales en el mercado de revistas/artículos considerados de mayor calidad y la emergencia de un mercado complementario liderado por editoriales -en 
algunos casos de dudosa procedencia (Truth, 2012) - que ofrecen acceso abierto a las publicaciones pero cobran a las y los investigadores por publicar. En la intersección de estos mercados, algunas revistas de aquel oligopolio editorial han comenzado a ofrecer la opción de publicación bajo acceso abierto, pero para ello cobran a las y los autores. De ese modo, el negocio de las editoriales académicas se mantiene, afectando la circulación del conocimiento en el primer caso, y reproduciendo las desigualdades en función del acceso a fondos para investigar en tanto quien tiene los fondos tendrá más facilidad para acceder a dicha publicación (Babini, 2011; Naidorf y Perrotta, 2017).

A nivel global, la ciencia considerada de "mayor calidad" suele ser justamente aquella que se publica en revistas que están bajo la órbita de grandes editoriales multinacionales. Seis grandes editoriales, cinco de ellas privadas con fines de lucro, controlan el $50 \%$ de las publicaciones científicas indizadas a nivel global, obteniendo márgenes globales de ganancia de casi un $40 \%$. El poder que ejercen estas grandes editoriales, como Elsevier o Springer, que manejan oligopólicamente el mercado de publicaciones científicas, fuerza a las universidades a pagar millones para acceder a un sistema de publicación altamente centralizado. En América Latina, en un contexto de estructural insuficiencia presupuestaria que se traduce en menores puestos de investigación, las presiones para publicar -principal criterio de evaluación para obtener un puesto- se acrecientan, lo que parece un contrasentido.

En este contexto, el acceso abierto, cuando no es privatizado ${ }^{3}$, es una iniciativa contrahegemónica que está ganando espacio. Asimismo, algunos países centrales (Francia, Alemania y Suecia) han cancelado sus contratos con algunas de esas editoriales oligopólicas debido a sus precios excesivos y buscan promover el acceso abierto a toda la investigación financiada con fondos públicos ${ }^{4}$.

El impacto de las grandes empresas editoriales sobre la investigación universitaria no sólo refiere a la limitación a la circulación pública del conocimiento y por lo tanto a su avance. Estas editoriales se transforman en reguladoras de cómo se produce conocimiento y en agencias de validación que son acríticamente asumidas como parámetro válido de conocimiento original. Dictaminan qué merece publicarse y qué no. Imponen criterios, reglas de juego y valores determinados por ellas mismas,

\footnotetext{
${ }^{3}$ La privatización del acceso abierto es un fenómeno que descompone el espíritu de esta herramienta que procura la democratización del conocimiento (sobre privatización del acceso abierto ver Naidorf y Perrotta, 2017).

${ }^{4} \mathrm{https}$ ://www.timeshighereducation.com/news/sweden-cancels-elsevier-contract-open-access-disputespreads
} 
como por ejemplo el factor de impacto, para incrementar su negocio. Adicionalmente, regulan la publicación de determinados temas y emiten señales sobre los temas preferibles a ser estudiados en la región.

Estos parámetros suelen ser usados por las instituciones científicas como criterios de calidad y tienen una influencia negativa en las políticas de evaluación de esta actividad y en la orientación y desarrollo de los proyectos que se llevan a cabo, especialmente en países periféricos. Atentan además contra la libre circulación de la información científica (CPS, 2018). En este sentido, los negocios en torno a la privatización del acceso abierto (Naidorf y Perrotta, 2017) y a las editoriales que lucran con el conocimiento primariamente financiados por los estados refuerzan la crítica a los criterios de evaluación primordialmente basados en la publicación científica. No pueden ser consideradores acríticamente como parámetro del buen desempeño de las y los académicos, cuestión que abordaremos en el apartado siguiente.

\section{CONCLUSIONES Y APORTES A LA REVISIÓN DE LOS CRITERIOS DE LA PONDERACIÓN DE LA PRODUCCIÓN CIENTÍFICA Y TECNOLÓGICA}

Los últimos veinte años fueron prolíficos en investigaciones sobre política científica en y desde América Latina ${ }^{5}$. Sin embargo, algunas tendencias arraigadas 0 poco problematizadas tienen un impacto significativo en las prácticas evaluativas que son orientadoras y performativas de prácticas y trayectorias académicas (Bianco, Mazzitelli y Tomassini, 2014). Se ha analizado cómo los investigadores en Argentina modifican sus lógicas de publicación científica a partir de los mecanismos de evaluación del CONICET (Beigel, 2017) y cómo los investigadores profundizan sus colaboraciones nacionales e internacionales en parte para incrementar su cantidad de publicaciones y así obtener mejores resultados en dichas evaluaciones (AguadoLópez, Becerril-García y Godínez-Larios, 2018).

Más allá de las investigaciones sobre criterios de evaluación, también han existido instancias que procuraron problematizar el impacto de la evaluación predominantemente basada en la cantidad de papers publicados (ver nota al pie 2). No obstante, este indicador se mantiene como el más relevante a la hora de valorar la calidad académica de un investigador o investigadora.

\footnotetext{
${ }^{5}$ Cabe reconocer la dinámica y la experiencia de los estudios latinoamericanos en ciencia, tecnología y sociedad que tuvieron lugar en la denominada Escuela de Pensamiento Latinoamericano en Ciencia y Tecnología, cuyo auge se manifestó durante las décadas del 60 y en los primeros años de los 70 y que fue discontinuado por las dictaduras militares, iniciadas por el golpe de estado en Chile en 1973 y ampliadas a toda América Latina a través del plan Cóndor.
} 
Aunque el frenesí de la publicación de artículos con referato parezca estar lejos de la satisfacción de necesidades privadas o de la contribución a la producción de innovaciones, en este trabajo apuntamos a poner en discusión cómo la masividad de publicaciones científicas y la búsqueda de publicación rápida de resultados contribuye a desvirtuar el valor de la calidad (cuando no la relevancia social de un tema de investigación) sin dar cuenta al mismo tiempo de la transferencia ciega de conocimiento. Asimismo, mostramos cómo estos artículos abordan temáticas que responden a agendas de investigación que satisfacen necesidades privadas, principalmente de empresas que concentran la propiedad intelectual y que se ubican en países centrales. Por otra parte, el crecimiento exponencial de publicaciones favorece el negocio editorial, también concentrado en pocas empresas de esos mismos países.

Definimos entonces a la ciencia privatizada como aquella que por medio de copiar agendas y prioridades exógenas al contexto de creación del conocimiento y a las necesidades sociales, beneficia la acumulación de ganancias privadas sin que quede ningún rédito de ese proceso para las instituciones productoras de ese conocimiento, ni tampoco por lo tanto para sus países de origen. La ciencia privatizada, tal como afirmamos anteriormente, utiliza como criterio primordial de evaluación del desempeño de las y los investigadores a los indexadores internacionales de cuantificación de artículos, y al factor de impacto de las revistas en donde éstos se publican como sinónimo de calidad académica. Desconoce así la existencia de un mercado editorial que no es reconocido como tal por las agencias de financiamiento de la investigación científica. También asociamos a la ciencia privatizada con la orientación mercantil de la investigación que se esfuerza más por obtener curas vendibles o aplicaciones de interés para las agendas de investigación internacionales dominadas por empresas multinacionales. Estas empresas constituyen monopolios intelectuales. Al priorizar sus agendas se deja de lado la prevención y la búsqueda de soluciones a los problemas que aquejan a nuestras sociedades en Latinoamérica. También se margina a la educación como ámbito de producción y transmisión de conocimiento, como instancia de validación social de los resultados de las investigaciones. A nivel más general, se deja de lado la pregunta acerca de para quién se produce conocimiento.

Actualmente, en función de criterios que resulten en las discusiones plenarias entre evaluadores menos equívocos y más comparables, estos prefieren contar 
papers. En especial, se valoran las publicaciones en revistas del circuito mainstream por sobre cualquier otro indicador de calidad académica o de perfil de investigador/a. Este criterio es el que prevalece a la hora de elaborar un orden jerárquico entre candidatos y candidatas en el cual una gran cantidad de aspirantes a investigadores e investigadoras de tiempo completo quedan expulsados del sistema dada la insuficiencia presupuestaria estructural de los países de la región y la inexistencia de ámbitos alternativos de inserción de las y los investigadores.

La falta de instancias para la definición de un perfil de investigador o investigadora y la ausencia de indicadores que permitan ponderar el impacto social juegan en contra de una orientación de la política científica que se considere autónoma y, al mismo tiempo, comprometida socialmente. En virtud de ello la propuesta que sigue busca sugerir una serie de indicadores alternativos apostando a que sean incluidos en la variable "producción científico-tecnológica" a la par del conteo de papers. Esta propuesta de nuevos criterios de evaluación busca estimular a la comunidad científica a la reflexión acerca del impacto económico, social y político de nuestro trabajo. Los indicadores propuestos son:

1. Ponderación del impacto social.

2. Consideración de áreas de vacancia.

3. Valoración del trabajo editorial (de editoriales locales).

4. Reconocimiento del trabajo con comunidades.

1. Las comisiones evaluadoras, usualmente compuestas por investigadores de gran trayectoria, no suelen atender ni valorar el impacto social de las investigaciones. Quizás por la falta de indicadores cuantitativos que faciliten su tarea de evaluación, el impacto social de una producción científica así como la relevancia social del tema de investigación son infravalorados.

2. Las áreas de vacancia han estado asociadas a temas estratégicos, aunque no lo son exactamente. En general, la vacancia es una categoría contextual y asociada a necesidades sociales regionales. Estas áreas suelen estar ausentes en las agendas de los países centrales que es donde se concentran los recursos para las investigaciones científicas de mayor envergadura. En este sentido, la vacancia reconoce un componente asociado a la relevancia social, aunque corresponde, como la etimología de la palabra lo refiere, a un tema poco desarrollado.

3. Tal como hemos afirmado, en parte la ciencia se vuelve privatizada porque se posiciona acríticamente frente al mercado editorial que asocia calidad con 
indexadores. De allí que la producción editorial propia y los esfuerzos locales por potenciarla no son bien ponderados entre las actividades de las y los investigadores locales. No existe ninguna valoración a la tarea editorial que, en general, está a cargo de las y los investigadores y sus universidades. Incluso la publicación en revistas de la propia universidad es considerada producción endógena y por lo tanto penalizada por las y los evaluadores.

4. Por último, proponemos valorar más el trabajo con las comunidades. No solamente nos referimos al trabajo de campo específicamente, sino a la intervención social, a la divulgación en el territorio, a la participación en los asuntos ciudadanos desde su experticia, a la contribución a la elucidación y respuesta a los grandes problemas nacionales y de la región que de una u otra manera se relacionan con los temas de investigación de cada quien.

Finalmente y a modo de cierre queremos afirmar que el intercambio con miembros de la comunidad académica local, con grupos de trabajo latinoamericanos ${ }^{6}$, la participación en múltiples instancias de evaluación académica, las reflexiones conjuntas con miembros de nuestro equipo de investigación cuyo objeto de estudio son algunos de los temas aquí abordados, nuestras tesis de doctorado y el intercambio con colegas de múltiples latitudes nos inspiran a procurar explicitar esta crítica y ser propositivas. Lo hacemos en el marco de la eterna y urgente pregunta sobre cómo y para quién hacemos ciencia en América Latina.

\section{REFERENCIAS}

Aguado-López, E., Becerril-García, A. y Godínez-Larios, S. (2018). Asociarse o perecer: La colaboración funcional en las ciencias sociales latinoamericanas. Reis, 161, 3-22.

Ahmadpoor, M., \& Jones, B. F. (2017). The dual frontier: Patented inventions and prior scientific advance. Science, 357(6351), 583-587.

Albornoz, M., Barrere, R. y Sokil, J. (2017). Las Universidades lideran la I+D en América Latina. En El Estado de la Ciencia 2017 (RICyT, pp. 31-44). RICyT.

\footnotetext{
${ }^{6}$ Como el denominado "La ciencia social politizada y móvil de una nueva agenda latinoamericana orientada a prioridades desde la universidad" (Grupo de Trabajo de CLACSO compuesto por miembros de 10 países).
} 
Arocena, R., Göransson, B., \& Sutz, J. (2015). Knowledge policies and universities in developing countries: Inclusive development and the "developmental university". Technology in society, 41, 10-20.

Arocena, R., \& Sutz, J. (2001). Changing knowledge production and Latin American universities. Research Policy, 30(8), 1221-1234.

Arocena, R., \& Sutz, J. (2010). Weak knowledge demand in the South: Learning divides and innovation policies. Science and Public Policy, 37(8), 571-582.

Babini, D. (2011). Acceso abierto a la producción científica de América Latina y el Caribe: Identificación de principales instituciones para estrategias de integración regional. CTS. Revista Iberoamericana de Ciencia, Tecnología y Sociedad, $6(17)$.

Beigel, F. (2010). Autonomía y dependencia académica: Universidad e investigación científica en un circuito periférico: Chile y Argentina, 1950-1980. Buenos Aires, Argentina: Editorial Biblos.

Beigel, F. (2017). Científicos periféricos, entre Ariel y Calibán. Saberes institucionales y circuitos de consagración en Argentina. Las publicaciones de los investigadores del CONICET. Dados, 60(3), 825-865.

Beigel, F., Gallardo, O., \& Bekerman, F. (2018). Institutional expansion and scientific development in the periphery: The structural heterogeneity of Argentina's academic field. Minerva, 56(3), 305-331.

Bekerman, F. (2010). Modernización conservadora: La investigación científica durante el último gobierno militar en Argentina. En F. Beigel (Ed.), Autonomía y dependencia académica. Universidad e investigación científica en un circuito periférico: Chile y Argentina (1950-1980) (pp. 198-220). Recuperado de https://bit.ly/2SXc9qY

Bianco, M., Mazzitelli, M. G. y Tomassini, C. (2014). Señales transmitidas por el sistema de fomento a la investigación. Tensiones en la orientación de la producción de conocimiento y las carreras académicas en Uruguay. Redes, 20(39), 159-182.

Buchbinder, P. (2005). Historia de las universidades argentinas. Buenos Aires: Editorial Sudamérica.

Codner, D. G., Becerra, P., \& Díaz, A. (2012). Blind Technology Transfer or Technological Knowledge Leakage: A Case Study from the South. Journal of technology management \& innovation, 7(2), 184-195. 
Codner, D. G., \& Perrota, R. M. (2018). Blind Technology Transfer Process from Argentina. Journal of Technology Management \& Innovation, 13(3), 47-53.

Confraria, H., \& Vargas, F. (2019). Scientific systems in Latin America: Performance, networks, and collaborations with industry. The Journal of Technology Transfer, 44(3), 874-915.

CPS: Cátedra Libre Ciencia, Política y Sociedad (2018). Publicaciones científicas, ¿comunicación o negocio editorial? Ciencia, Tecnología y Política, 1(1). DOI: https://doi.org/10.24215/26183188e005

Dávila, M. (2006). Vinculación universidad-sector productivo-estado en la Facultad de Agronomía de la Universidad de Buenos Aires: la apertura al medio como eje de transformación institucional. En S. Llomovatte, F. Juarros, J. Naidorf y A. Guelman (Eds.), La vinculación universidad-empresa: Miradas críticas desde la universidad pública. Buenos Aires: Miño y Dávila.

Durand, C., \& Milberg, W. (2019). Intellectual monopoly in global value chains. Review of International Political Economy, 1-26. DOI: https://doi.org/10.1080/09692290.2019.1660703

Dutrénit, G., \& Arza, V. (2010). Channels and benefits of interactions between public research organisations and industry: Comparing four Latin American countries. Science and Public Policy, 37(7), 541-553.

Iñigo Carrera, J. (2007). La formación económica de la sociedad argentina. Volumen I, Renta agraria, ganancia industrial y deuda externa. 1882-2004. Buenos Aires: Imago Mundi.

Juarros, M. F. (2006). La vinculación universidad-empresa-sector de la producción de bienes y servicios, vista por los docentes investigadores. El caso de la Facultad de Farmacia y Bioquímica de la Universidad de Buenos Aires. En S. Llomovatte, F. Juarros, J. Naidorf y A. Guelman (Eds.), La vinculación universidad-empresa: Miradas críticas desde la universidad pública (pp. 171-212). Buenos Aires, Argentina: Miño y Dávila.

Juarros, M. F. y Martinetto, A. B. (2008). Limitantes del investigador académico: Financiamiento y políticas científicas. Nómadas (Col), 29, 50-63.

Llomovatte, S. (2006). Para una crítica del modelo de la triple hélice: universidad, empresa y estado. En S. Llomovatte, F. Juarros, J. Naidorf y A. Guelman (Eds.), La vinculación universidad-empresa: una crítica desde la universidad pública (pp. 21-42). Buenos Aires: Miño y Dávila. 
Naidorf, J. (2006). Antecedentes de la vinculación universidad-empresa desde la perspectiva del cambio en la cultura académica (1955-1984). En S. Llomovatte, F. Juarros, J. Naidorf y A. Guelman (Eds.), La vinculación universidad-empresa: Miradas críticas desde la universidad pública (pp. 91-112). Buenos Aires: Miño y Dávila.

Naidorf, J. (2009). Los cambios en la cultura académica de la universidad pública. Buenos Aires: EUDEBA.

Naidorf, J. y Perrotta, D. (2017). La privatización del acceso abierto. Nuevas formas de colonización académica en América Latina y su impacto en la evaluación de la investigación. Universidades, 73, 41-50.

Navarro, J. J. y Quesada, F. (2010). El proyecto Camelot (1964-1965). La dependencia académica, entre el escándalo y el mito. En F. Beigel (Ed.), Autonomía y dependencia académica. Universidad e investigación científica en un circuito periférico: Chile y Argentina (1950-1980) (pp. 145-167). Buenos Aires, Argentina: Editorial Biblos.

OECD (2012). Programme on Innovation, Higher Education and Research for Development. Research Universities: Networking the Knowledge Economy. Boston.

Orhangazi, Ö. (2018). The role of intangible assets in explaining the investment-profit puzzle. Cambridge Journal of Economics, 43(5), 1251-1286.

Pagano, U. (2014). The crisis of intellectual monopoly capitalism. Cambridge Journal of Economics, 38(6), 1409-1429.

Rikap, C. (2017). The corporization of a public university with free undergraduate education: Endangering autonomy at the University of Buenos Aires. World Social and Economic Review of Contemporary Policy Issues, 8, 44-59.

Rikap, C. (2018). Innovation as Economic Power in Global Value Chains. Revue d'Économie Industrielle, 163, 35-75.

Rikap, C. (2019). Asymmetric Power of the Core: Technological Cooperation and Technological Competition in the Transnational Innovation Networks of Big Pharma. Review of International Political Economy, 26(5), 987-1021. DOI: https://doi.org/10.1080/09692290.2019.1620309 
Rikap, C., \& Harari-Kermadec, H. (2019). Motivations for collaborating with industry: Has public policy influenced new academics in Argentina? Studies in Higher Education, 1-12. DOI: https://doi.org/10.1080/03075079.2019.1659764

Riquelme, G. C. y Langer, A. (2013). Los docentes universitarios y la producción y circulación del conocimiento: Un estudio sobre universidades argentinas. CIANRevista de Historia de las Universidades, 16(1), 81-114.

Sutz, J. (2000). The university-industry-government relations in Latin America. Research policy, 29(2), 279-290.

Tijssen, R. (2019). On science industry linkages worldwide. Presentado en The 13th Workshop on The Organisation, Economics and Policy of Scientific Research, Bordeaux.

Truth, F. (2012). Pay big to publish fast: Academic journal rackets. Journal for Critical Education Policy Studies, 10(2), 54-105.

Unzué, M. (2013). Autonomía, evaluación y políticas públicas. Tendencias y límites en los sistemas de Argentina y Brasil. En M. Unzué y S. Emiliozzi (Eds.), Universidad y políticas públicas. En busca del tiempo perdido (pp. 9-48). Buenos Aires, Argentina: Imago Mundi.

Vessuri, H. (1987). La revista científica periférica. El caso de Acta Científica Venezolana. Interciencia, 12(3), 124-134.

Wang, L., \& Li, Z. (2019). Knowledge flows from public science to industrial technologies. The Journal of Technology Transfer, 1-24. DOI: https://doi.org/10.1007/s10961-019-09738-9 